\title{
A Driving Support System Base on Traffic Environment Analysis
}

\author{
Yang Shuo and E-Jung Choi* \\ Department of Multimedia, HanNam University, Republic of Korea; \\ yangshuo100@hotmail.com, donghyunk1986@gmail.com
}

\begin{abstract}
Objectives: Vehicle driving are complex action, when drivers couldn't focus on driving the accident will happen at any moment, we build a system to help driver prevent from traffic accident. Methods/Statistical analysis: In this paper we build a system based on computer vision techniques using support vector machine training detection pattern include distance computing and alert module. At this research we use visual data instead traditional ultrasonic wave data as source data because visual data will provide more environment detail and range than sound wave can, so using the visual data make long rang driving environment alert possible. Findings: In this research we using Haar-like features as system detection feature, because Haar-like feature is a fast and stable detection feature, in the followed test this algorithm total fit our system requires. After small sample Haar-like feature test we use Support Vector Machine to training large sample for system detection pattern and it include 3 parts: pedestrian, vehicle and motorcyclist. Base on driving video sample analysis we found there's a "hot zone" in camera that most of valuable target will appear in this area, it improved our system computing effectiveness. Application/Improvements: In research we achieve our goal, system can help driver handing complex driving environment to avoid accident happen, future research we will focus on detection accuracy and distance accuracy.
\end{abstract}

Keywords: Computer Vision, Driving Support, Object Detection, Traffic Environment Computing

\section{Introduction}

Modern transportation enhanced human society's development greatly, but the transport vehicles become more danger and deadly to humans self. Vehicle driving is complex behavior; lots of reason can affect driver's implementation performance, its base on driver's experiences, physical conditions (tiredness, injuries) and external environment (weather, vehicle conditions). For reduce the number of traffic accident, There's two ways to achieve the goal internal and external. Internal parts are base on vehicle driver and external is about driving environment it's include vehicle conditions and driving environments. In the external part we found there's no room for improve if vehicle owner follow their vehicle inspection schedule and weather forecast, so we change our research target to internal part. Usually vehicle driver can make right decision base on their experiences and knowledge to take reaction to environment quickly, but drivers are human they could make wrong decision or mistake cause an accident should have avoided. In order to reduce that kind traffic accident we design and build a driver support system base on driving environment.

\section{System Design}

In this paper, we design and build a system use for improving vehicle driving safety. First of all we need to know which kind accident happens frequently, from Korea ministry of transportation's traffic accidents statistics most accidents happened between vehicle and vehicle (72.5\%), but when accident happened between vehicle and people (total accident's $25.5 \%$ ) its mortality (3.66\%) are higher than other kind accident (vehicle vs. vehicle $1.18 \%)$. so we selected people and vehicle as our main detecting targets. In our system's objects detection part we

*Author for correspondence 
using wavelets as detection factor, because wavelet based object detection can working in noisy backgrounds\$, in real driving situations background noise exist everywhere using wavelet factor can improve our system's detection accuracy. As Figure 1 indicates, in our system we selected several kind vehicles as vehicle pattern, the people detection part we using pedestrian and motorcyclist as detection pattern.

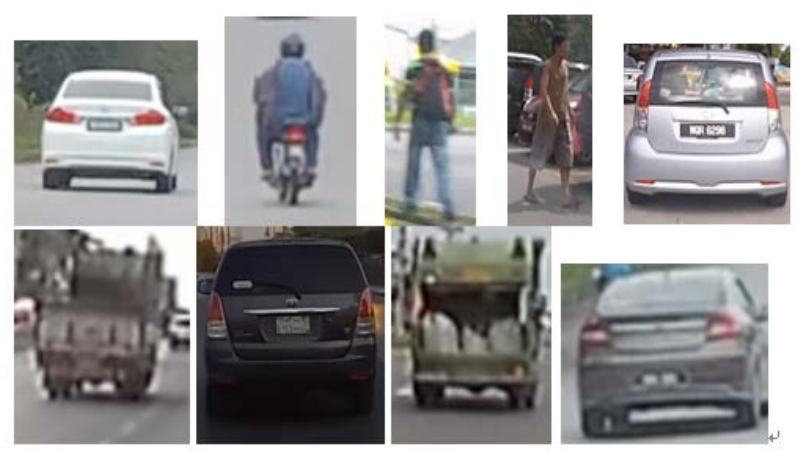

Figure 1. Traning samples.

\subsection{Target Detection}

In order to create detection module we using Support Vector Machines (SVM) as machine learning method because it can avoid over-fitting 阳, that's very important for our project, because vehicle have several kind include lots of model. Follow the reason we explained at previous chapter, we using vehicle, pedestrian and motorcyclist's Haar-like features as our system's detection features.

\subsubsection{Haar-Like Features and Viola-Jones Framework}

In our system, target object always keeping in dynamic, so the target feature must be simple for computing to get result quickly and reliability. Harr-like features are digital image features because its intuitive similarity with Harr wavelets so they get their name. Viola and Jones provide a fast detection framework for face detection. it can detection which minimizes computation time while achieving high detection accuracy, its fit our goal, because our system will active in a complicated object environment both accuracy and computing speed are we needed. Its high speed computing are base on summed area tables/, which also called integral images, it can be defined as two-dimensional lookup tables in the matrix form with the same size of the original image. The integral image's descriptions are followed:
$S U M=I(C)+I(A)-I(B)-I(D)$

Follow the Figure 2, points A,B,C,D belong to the integral image $\mathrm{I}$, if we want get shaded rectangular area's sum, we need use C area and remove area $A, B$ and $C$, in description (a) there not only remove the area $B$ and D's sum, it also plus A area's sum that's because area B, C, D are include area $\mathrm{A}$, so we need plus $\mathrm{A}$ one more time for remove area $B$ and C's effect.

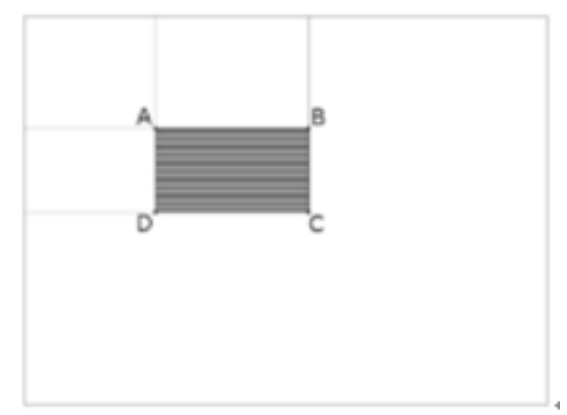

Figure 2. Integral images.

In Viola-Jones object detection framework the algorithm has four stages:

1. Haar Feature Selection

2. Creating an Integral Image

3. Adaboost Training

4. Cascading Classifiers

The Viola-Jones framework are design for human face detection as shown in Figure 3, its one goal classifier system, but our system is multiple goal classifier system, so in our research we only use stage 1 and 2 the framework's feature parts.

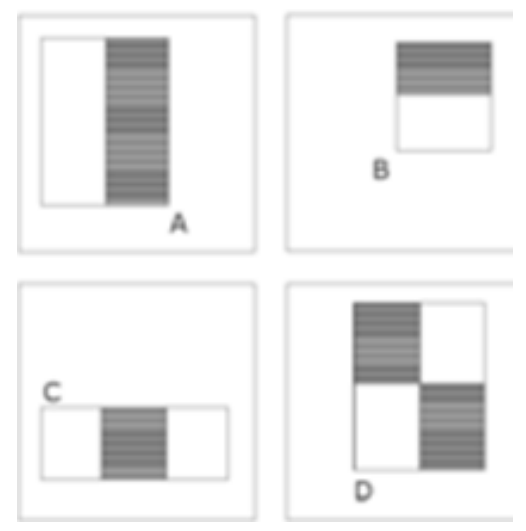

Figure 3. Four feature types used by Viola and Jones. 
The feature types Viola-Jones used are for face detection, but we found the feature pattern of organs on face can use in vehicle features in Figure 4.
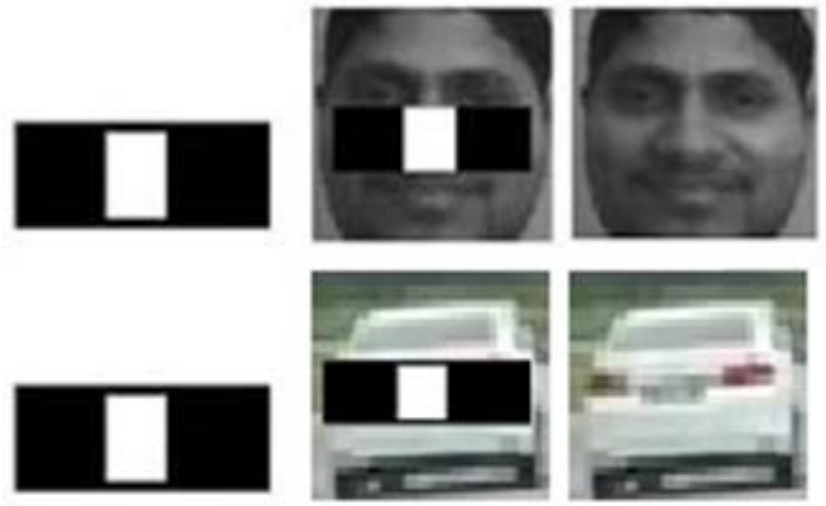

Figure 4. Haar-like feature sample: human face and vehicle.

In the test we get great result of vehicle detection. The detection result of pedestrian and motorcyclist also has good result as shown in Figure 5.
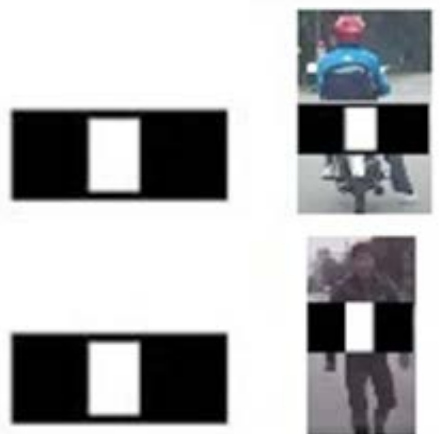
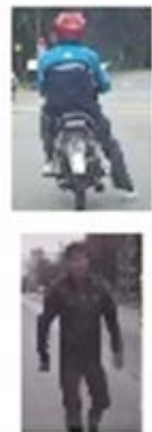

Figure 5. Haar-like feature sample: motorcyclist and pedestrian.

When we finished feature sample test and get enough sample image, the next step is training a classifier for our system.

\subsubsection{Support Vector Machine Classifier}

In our research we have 3 goal targets, so if we use Adaboost as classifier will cause low performance to system and avoid over-fitting. So we decided use SVM as our system classifier because SVM can separate object in different space.
In Figure 6 the vehicles (blue) are separate nicely with motorcyclist (black) and pedestrian (red), but between motorcyclist and pedestrian separate result are slight crossed, that's because in the image those two have similar shape.

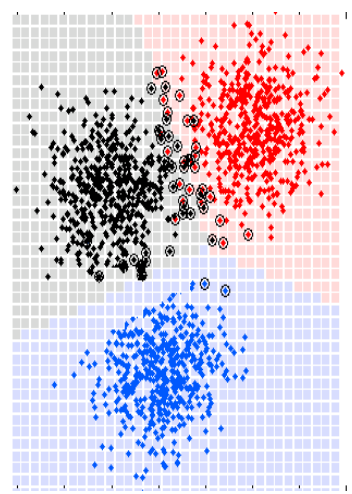

Figure 6. SVM classifier of training samples, red - pedestrian, black - motocyclist, blue - vehicles.

\subsection{Distance Data Computing}

Before we calculate target's distance we need create a list to save it in, if there's no list we can't confirm it is same target we tracked. When the goal targets detected we need check the target existed in our tracking list. In our case we using histogram as tracking factor, its fast enough when running multiple object tracking], when target was detected, first check it existed in histogram list or not, if not in list create a new list item and store it in the new item.

\subsubsection{Target Object Tracking List}

For tracking target object we made a list to record target information, the list include object ID, position in camera, relative distance and histogram data. First part is ID, for object ID number's uniqueness, object ID is the sum of detected object's histogram when it been detected in first time. Second is position in camera, we record detected object's center point for calculate object distance change, it always last time position in camera. Relative distance is the distance to camera's define position, because camera's position in vehicle are different the define position also different in each camera. Last one is object's histogram, it's the most important information in list, we use it define is it the same vehicle in list. Every camera has a vanishing point base on it we can set a "hot zone" of camera.

In our research most target object are detected in "hot zone" ( $74.7 \%$ in total detected object) Figure 7 , so 
we set "hot zone" as primary detection area, when we got object's detection area now we can track object easily. Our tracking system base on Porikli and Tuzel's research in our case between camera and target both are moving object, and target object's appear position are predictable. In Porikli's research $F$ be the $W \times H \times d$ dimensional feature image extracted from $I$.

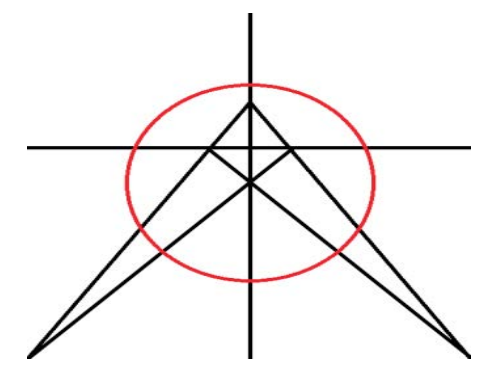

Figure 7. "Hot Zone" in camera (red circle area - valuable target appear area).

$\mathrm{F}\left(\mathrm{x}, \mathrm{y}_{-}=\Phi(\mathrm{I}, \mathrm{x}, \mathrm{y})\right.$

Where the function $\Phi$ can be any mapping such as color, image gradients ${ }^{9}$, in our case $\Phi$ is the detected object histogram, because our detection computing already gives position information we need so we need just compare the new frame object with the last frame object. If the object tracking feature are fit our object feature threshold then update object list item feature date, if the object feature value are lower than feature threshold then add a new item in list. If an object are lost tracking, the item Time To Live (TTL) will minus 1, when TTL gone 0 list item will delete, normally we set TTL as 120 (5 second $\times$ camera fps).

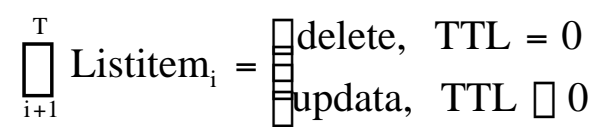

Where the $\mathrm{T}$ is list item total number, it will change when list item add or delete.

\subsubsection{Target Object Distance}

When we driving a vehicle, we can't know other vehicles distance exactly between us and others. But we can rough estimate the distance between us and target, normal the vehicle's width are smaller than road lane, this time we use the Europe road design as vehicle and pedestrian data as shown in Figure 8.

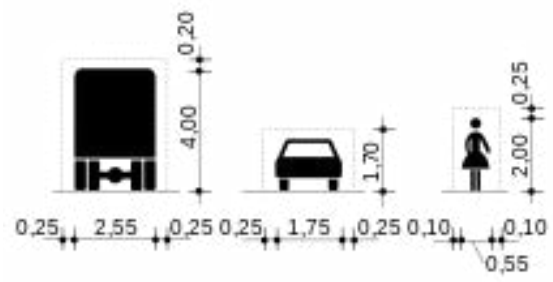

Figure 8. Widths and heights in road design of Europe (From left to right: truck, car and pedestrain).

In our system, we only use vehicle's width data, because there's huge different between car and trucks, so we define all kind of vehicle's width are 2.15 meter, so when target was detected, at same time we can estimate target's distance. Via angular mil, the target object distance can get through calculating.

$\mathrm{D}=\frac{\mathrm{S}}{\mathrm{mil}}$

Where $\mathrm{D}$ is distance to target in kilometers, $\mathrm{S}$ is actual size of target in meters; mil is the observed angle of view of target measured in Mil-dots. In our case there's no need military level accuracy, so we change the above formula:

$$
D=\frac{W_{\text {vehicle }}}{W_{\text {camera }}}
$$

Before we set device up, we use camera take several sample of vehicle, to make a standard for system camera for example: we use system camera get vehicle display width in different distance, because the vehicle width change in camera are linear, so we compare vehicle's width (in this case $2.15 \mathrm{~m}$ ) with the vehicle width in camera, we using this way to get test camera's lens property.

If detected targets in the tracking list then compare its distance with least distance which saved in storage space. If its distance or relative velocity change rate higher than alert threshold then trigger alert signal.

\section{Test Design and Result}

\subsection{Test Design}

For test our system, we set system on a family car and driving in city road. We running system test at 10:30 am in a day with clean weather, driving distance are approximate 23 kilometers. In the test the vehicle driving average speed is $52 \mathrm{KM} / \mathrm{H}$. 


\subsection{Test Result}

The test data include two parts, first part is to test object detection function and get systems correct detection rate Figure 9, second part is test distance calculate function's effectiveness. In the process of test there has 834 records, include 796 vehicle detection records and 34 pedestrians detection records and 4 motorcyclist detection records received. In testing process there have 47 goal targets missed and 28 false detections as show in Table 1.

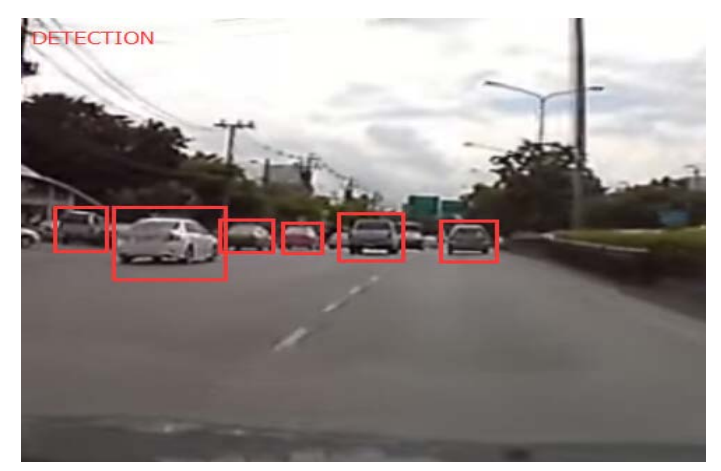

Figure 9. Detection processing(red box - detected vehicle).

Table 1. Test result of target detection

\begin{tabular}{lccc}
\hline Targets & $\begin{array}{c}\text { Detection } \\
\text { Record }\end{array}$ & $\begin{array}{c}\text { Validity } \\
\text { Detection }\end{array}$ & $\begin{array}{c}\text { Miss } \\
\text { Detection }\end{array}$ \\
\hline Vehicles & 796 & 772 & 28 \\
Pedestrians & 34 & 31 & 15 \\
Motorcyclists & 4 & 4 & 4 \\
\hline
\end{tabular}

In distance computing part shown in Figure 10 we found when traffic lights turning red the distance between target and us will reducing quickly, so this effect could make false alert frequently, in other situation this system operation was good.

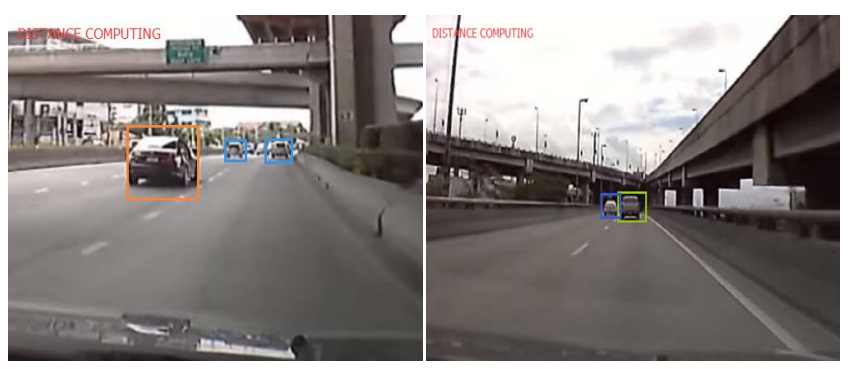

Figure 10. Distance computing result (blue - long distance, green - medium distance, orange - short distance).

\section{Conclusions}

In this paper we provided a driving support system based on environment analysis by using wavelet transform and SVM.
From test results our system has been shown to be effective in detecting goal targets and calculate vehicle distance. But this system still has several problems to solve, first is detection accuracy, in the driving test where a lot of miss and fault detection result, especially in pedestrian and motorcyclists part. The other is distance false alert, because vehicle driving are not running in uniform velocity speed, especially when vehicle passing crossroads or waiting traffic signals.

Further work will be focused on system's shortage, include detection accuracy and alert accuracy, because this system still in prototype stage, the system algorithm and structure have a huge space that can be improved and optimized.

\section{Acknowledgment}

This paper was supported by 2016 HanNam University Research Fund.

\section{References}

1. Korea Ministry of Transportation. Traffic Accident Summary 2015 [Internet]. 2016 [cited 2016 Jun 12]. Available from: http://www.molit.go.kr/USR/policyData/m_34681/ dtl.jsp?id=3992.

2. Strickland RN, Hee IH. Wavelet transform methods for object detection and recovery. Institute of Electrical and Electronics Engineers (IEEE) Transactions on Image Processing. 1997 Jun; 6(5):724-35.

3. Brown MP, Grundy WN, Lin D, Cristianini N, Sugnet CW. Knowledge-based analysis of microarray gene expression data by using support vector machines. Proceedings of the National Academy of Science. 2000; 97(1):262-67.

4. Viola P, Jones M. Rapid object detection using a boosted cascade of simple features. Institute of Electrical and Electronics Engineers (IEEE) Society Conference on Computer Vision and Pattern Recognition (CVPR). 2001; 1:1-9.

5. Viola P, Jones M. Robust real-time object detection. International Journal of Computer Vision. 2004; 57(2):137-54.

6. Crow F. Summed-area tables for texture mapping. Computer Graphics, SIGGRAPH. 1984 Jul; 18(3):207-12.

7. Corinna C, Vapnik V. Support-vector networks. Machine learning, SPRINGER. 1995; 20:273-97.

8. Bernardin K, Elbs A, Stiefelhagen R. Multiple object tracking performance metrics and evaluation in a smart room environment. In the Sixth Institute of Electrical and Electronics Engineers (IEEE) International Workshops on Visual Surveilance, Graz, Austria; 2006 May. p. 1-8.

9. Porikli F, Tuzel O, Meer P. Covariance tracking using model update based on lie algebra. Institute of Electrical and Electronics Engineers (IEEE) Society Conference on Computer Vision and Pattern Recognition (CVPR). 2006 Jun; 1:728-35. 